\title{
The structure and properties of materials used in advanced drug delivery systems
}

\author{
KAREL PETRAK \\ CIBA-GEIGY Pharmaceuticals, Advanced Drug Delivery Research Unit; Wimblehurst \\ Road, Horsham, West Sussex RH12 4AB, England
}

\begin{abstract}
The various requirements placed on materials that might serve as suitable components in drug delivery systems are discussed. Special attention is paid to the interactions between the drug delivery constructs and the biological environment, using the interactions in the vascular compartment of the body as an example.

Two alternative general approaches are compared: (a) "controlled drug release", which aims to reduce or eliminate side effects by producing a steady therapeutic concentration of drug in the body; (b) "site-selective drug delivery", which aims to ensure that the drug is delivered to the site of its biochemical and disease-related site of action, at the same time maintaining the drug inactive elsewhere in the body.

It is concluded that materials for delivering drugs to selected sites of disease within the body must be designed to utilise the unique features (structure, function, rhythm) of the main elements involved in the disease. Equally important is to design drug carriers that do not interact non-specifically within the body so that their specific action would be prevented. It is difficult to see how this could be achieved using materials entirely "foreign" to the body. Utilising the primary structures used by the biological systems (proteins, glycoproteins, carbohydrates) and creating novel higher structures (secondary, tertiary, quartenary) that mimic the native material is the logical way forward in the search for new drug delivery systems, and we need to turn more and more to the molecular basis of biology for guidance and inspiration.
\end{abstract}

Keywords. Controlled release; site-specific drug delivery; endothelial transport of macromolecules; drug carrier-cell interactions.

\section{Introduction}

The purpose of this presentation is to discuss the various requirements placed on materials that might serve as suitable components in drug delivery systems. Special attention will be paid to the interactions between drug delivery constructs and the biological environment, using the interactions in the vascular compartment of the body as an example.

\section{Drug delivery-purpose and rationale}

Traditionally the main line of pharmaceutical research has been to discover new compounds with biological activity that could be utilised in the treatment of diseases. There are several main disadvantages associated with the use of most conventional drugs. Such drugs are distributed throughout the body according to their physical properties such as solubility, partition coefficient, charge. In consequence, drugs can reach a variety of sites at which the drugs may be inactive, or where their action is either desirable or harmful, or both. Consequently, unwanted side-effects may result.

In many cases, the drug may be toxic to normal cells, and significant damage to 
healthy cells can result. Both the therapeutic and the harmful/toxic effects depend on the concentration of the drug at various sites in the body. Within the optimal therapeutic range (figure 1) the therapeutic effects of the drug outweigh its harmful action. At concentrations outside this range the drug is either not active (at low concentrations) or its harmful effects become prominent.

There are currently two distinct approaches to improving the drug action via its mode of delivery.

a) Controlled drug release: This aims to reduce or eliminate side effects by producing a steady therapeutic concentration of drug in the body. This is achieved through zero-order release of active drugs, and does not usually lead to a changed distribution of the drug in the body as compared to the distribution resulting from a conventional, intermittent drug dosage.

The mode and the rate of input of drug into the body can and has been controlled directly using various infusion and pumping devices. This mode of delivery will not be discussed here, instead we shall consider only diffusion- or fluxbased systems.

Drugs can enter the body by crossing the epithelium after being released from systems placed "outside" the body. A typical example of this is the transdermal drug delivery (figure 2). This type of device can establish a concentration gradient

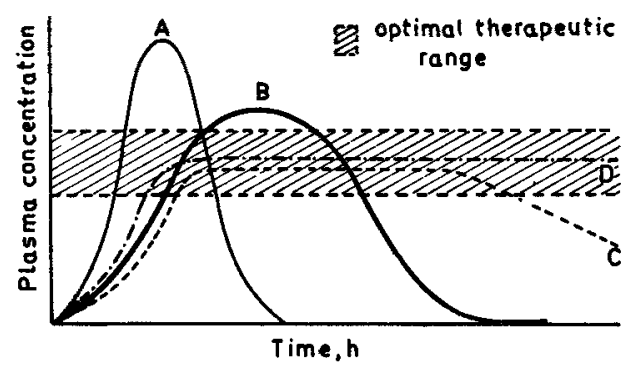

Figure 1. Plasma concentration curves resulting from the use of conventional tablet or capsule (A), slow-release preparation (B), OROS tablet (C), and transdermal patch (D) (Documenta CIBA-GEIGY 1987).

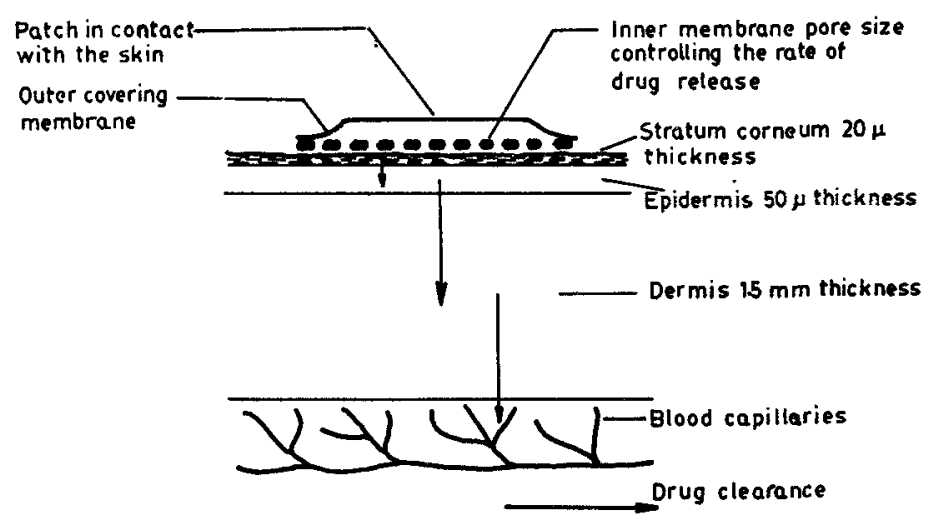

Figure 2. The structure and function of a transdermal patch (Documenta CIBA-GEIGY 1987). 
from the drug reservoir in the patch, through the skin to which it is applied, and the body. The skin is a body organ constructed to keep out harmful substances, bacteria and viruses, and the rate of transport of drugs across the skin is generally low. The rate-limiting step is usually given by the transport across the stratum corneum, and substances with a molecular weight above 1,000 are unlikely to be absorbed through intact skin. The transdermal devices are therefore suitable for drugs that are active in the body at very low dosage, have a short residence half-life and a high lipid-water partition coefficient. Systemic transdermal drug delivery is limited to around $10 \mathrm{mg}$ of active agent per day. An advantage of this delivery results from the fact that skin absorption avoids the "first pass" effect of hepatic metabolism to which most orally administered drugs are exposed. The practical problems that remain to be solved are how to enhance the flux of drug through the skin (e.g. via the use of various inert solvents), and to develop strong adhesives that would not elicit any skin reaction.

OROS is an example of an oral delivery system that achieves a constant rate of release of active substance over a predictable period of time (figure 3). Water from the gastrointestinal tract is driven by osmosis through a semi-permeable membrane of the device. The active substance cannot pass through the membrane but is forced out of the device by the resulting hydrostatic pressure through the release opening.

The delivery systems located "outside" the body do not of course interact in a significant way with the defence (immune, phagocytic) mechanisms of the body. Most implantable or injectable controlled release systems again modulate the drug availability by regulating its diffusion out of a "solid" material. Biodegradation of such materials can provide an additional parameter that can influence the overall rate of drug release. More significantly, the body will respond to the presence of a "foreign" material in an attempt to remove it (figure 4). Such body reaction is mediated by cellular and non-cellular interactions of the body with the drug delivery system, and will determine whether the particular system is ultimately suitable for therapy. For example, drug-loaded collagen sponge implants have been used to enhance healing in osteochondral defects (Speer et al 1980). Foreign body

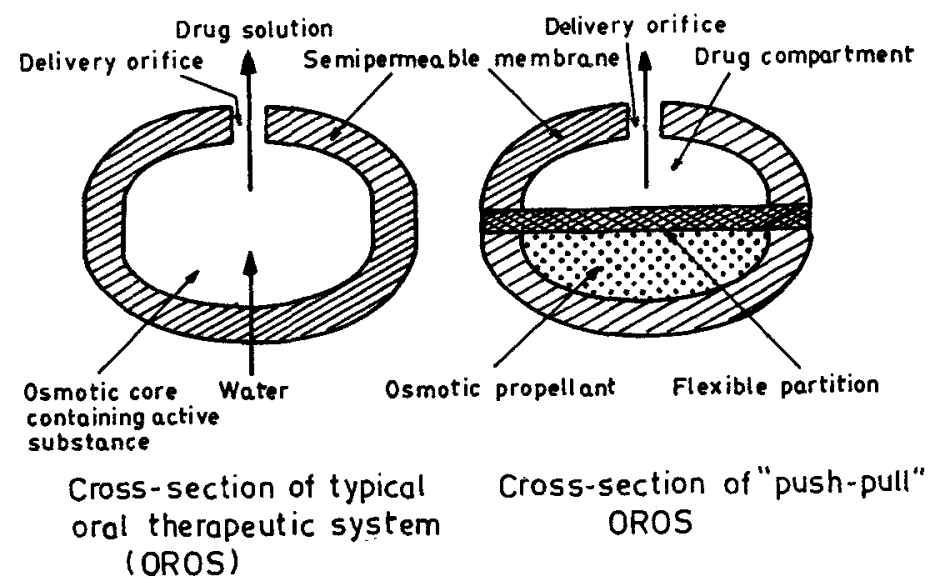

Figure 3. The structure and function of the OROS tablet (Documenta CIBA-GEIGY 1987). 


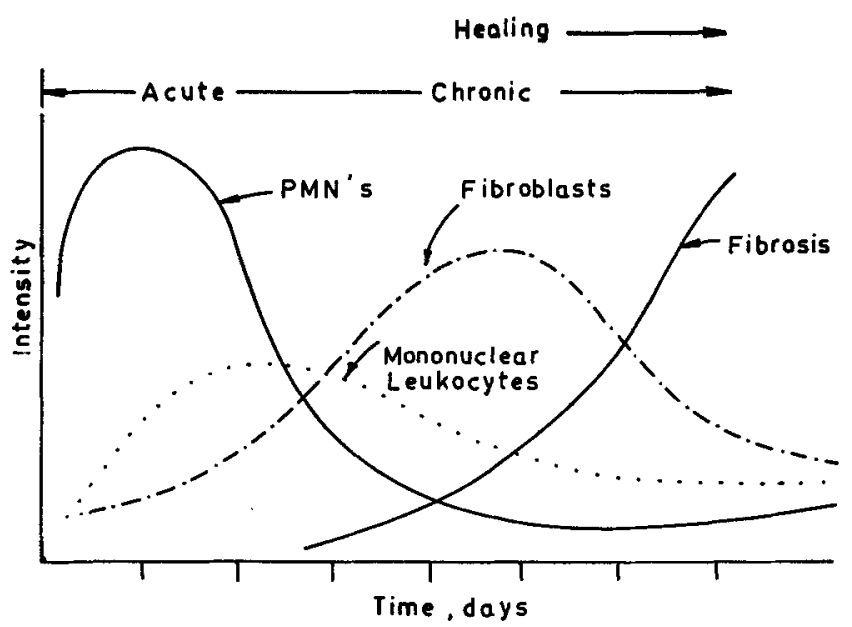

Figure 4. The nature and timing of the possible inflammatory response to a drug-delivery system.

giant cell reactions to the implanted sponge have been correlated however with cytotoxic effects upon fibroblasts in tissue culture. Observations indicated that leaching of glutaraldehyde used in the sponge to control the properties of collagenbased structures was sufficient to produce adverse cellular effects.

Ideally, various body responses could be used constructively as triggering events for releasing drugs. For example, the rate of release of a drug from a polymer matrix can be varied by swelling and shrinking the polymer in response to $\mathrm{pH}$ changes. When the matrix also contains an enzyme the action of which results in a local change of $\mathrm{pH}$ (e.g. glucose oxidase responding to sugar level), the swelling of the polymer and thus the release of drug (e.g. insulin) can be induced by the local sugar levels in the body. Similarly, penicillin amidase has been immobilized in water-soluble nonstoichiometric polyelectrolyte complexes (Margolin et al 1983). The particles of such complexes can undergo a cooperative solution-precipitate phase transition as a result of small changes in $\mathrm{pH}$. The activity or the enzyme contained within the complex can thus be regulated as it is influenced by the state of the matrix.

b) Site-selective drug delivery: This aims to ensure that the drug is delivered to the site of its biochemical and disease-related site of action, at the same time maintaining the drug inactive elsewhere in the body.

There is no doubt that designing drugs specifically to perform a precise biological function as it relates to a disease, is a sound principle to follow. The desired function of the drug cannot however be separated from its access to, and its retention at its site of action. Considerations of the rate and mechanism of transport, and therefore of the administration to, and the delivery within the body, must form a part of overall drug design.

Many specific processes in the body utilise the transport of macromolecules across biological membranes (endocytosis). It is then natural to consider this process for controlling the movement of drugs in the body via the use of 


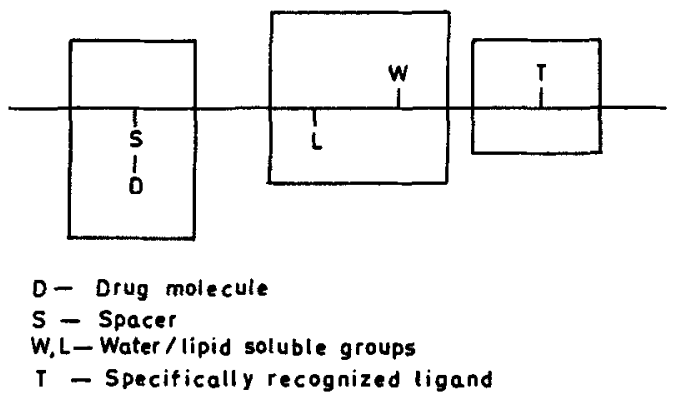

Figure 5. Compartmental model of a polymeric drug carrier.

macromolecular carriers (figure 5). Such carriers must be prepared in a reproducible way (e.g. with uniform size distribution, in a pure form), must have drug attachment/release sites, must be able to change the drug distribution in the body, must not elicit unwanted body responses (e.g. inflammation), and must be biodegradable.

Here we want to examine the events that might lead to the removal of the carrier from circulation.

(i) Transport across the endothelium-Carriers can leave the vascular compartment by crossing the biological barrier that separates the blood compartment from the rest of the body, i.e. the endothelium. For synthetic carriers this occurs by a passive transport, therefore the movement of the carrier depends on the fluid fluxes prevailing in any particular endothelium, with the rate of transport depending on the size of the carrier. In general, for the normal endothelium, as the size of the carrier approaches $90 \AA$, the rate of transport approaches zero. After crossing the renal endothelium, the carrier is removed from the body by filtration through the kidneys (here the size cut-off is close to $50 \AA$ ) (figure 6). The movement of the carrier across the endothelium in other organs will determine the availability of the carrier, and therefore of the drug at the selected sites outside the vascular compartment.

(ii) Interactions of the carrier with the components of the vascular compartmentFor a carrier to have an opportunity to access other sites within the body, it needs to avoid being recognised, and removed by the mononuclear phagocytic (reticuloendothelial) system (MPS or RES, respectively). It is a serious drawback of all carriers developed so far (e.g. colloidal particles, liposomes, drug-modified polymers) that they tend to be removed rapidly from circulation by the organs of the MPS (mainly the liver, spleen, bone marrow). It is believed that the event leading to this removal is so-called opsonisation, which involves adsorption of proteins to the surface of the carriers. In trying to establish what material properties are required from synthetic carriers to avoid the MPS uptake, we can take solace from the fact that several naturally occurring "particles" having different chemical composition and different sizes, have nevertheless an extended circulating half-life in the body (table 1).

It is also interesting to realise that foreign organisms that can invade the body and survive (bacteria, viruses) structure their surface to resemble the structures considered by the defence systems of the body as "normal".

Attempts have been made for many years now to alter the chemical structure of the surface of colloidal particles. Increasing the hydrophilicity of the surface, 


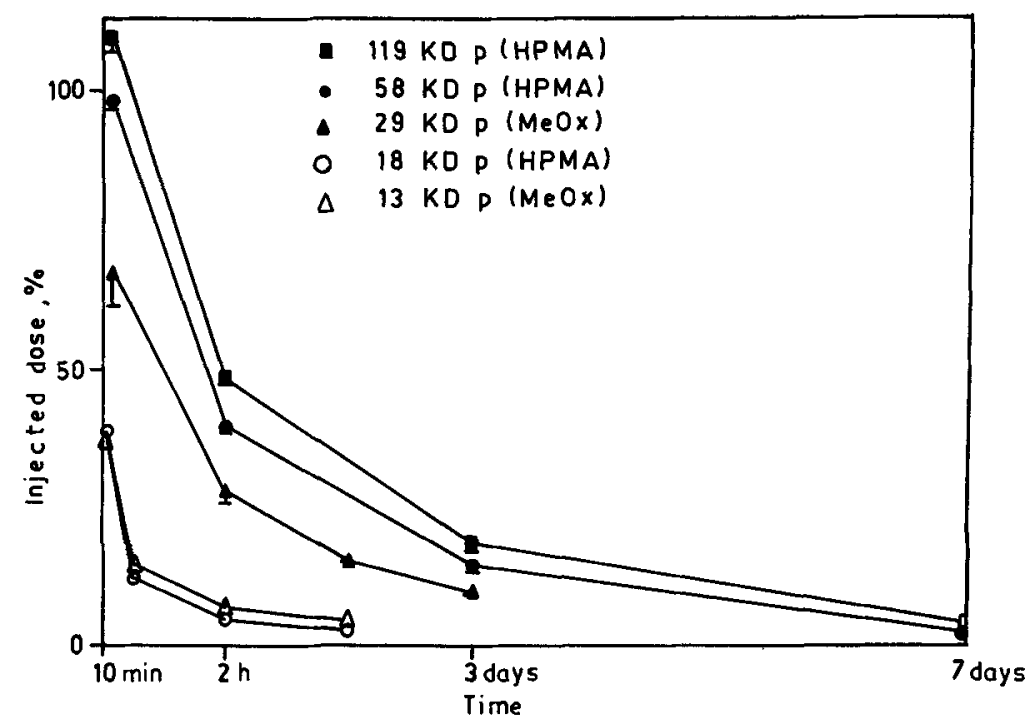

Figure 6. Blood levels of water-soluble polymers during seven days after intravenous administration (HPMA-poly (2-hydroxypropylmethacrylamide); MeOx-poly (methyloxazoline)).

Table 1. Sizes and circulating half-lives $\left(t \frac{1}{2}\right)$ of some natural particles.

\begin{tabular}{llc}
\hline Substance & $\begin{array}{c}\text { Size } \\
(\mathrm{nm})\end{array}$ & $\begin{array}{c}t \frac{1}{2} \\
\text { (days) }\end{array}$ \\
\hline $\begin{array}{l}\text { Albumin } \\
\text { Low-density } \\
\text { lipoproteins }\end{array}$ & 15 & 19 \\
$\begin{array}{c}\text { High-density } \\
\text { lipoproteins }\end{array}$ & $17-25$ & $3-4$ \\
$\begin{array}{c}\text { Red blood cells } \\
\text { (erythrocytes) }\end{array}$ & $7-12$ & $5-6$ \\
\hline
\end{tabular}

together with rendering the surface negatively charged under the physiological conditions, does tend to increase the survival time of particles in circulation. The success to date, however, has been limited. For example, with liposomes half-lives not higher than $20 \mathrm{~h}$ have been achieved so far.

Perhaps the best results in this direction has been obtained with grafting polyoxyethylene chains to proteins (Abuchowski et al 1977). When foreign enzymes, e.g. L-asparaginase, urate oxidase, are administered to humans, the enzymes are rapidly removed from circulation. Modifying such proteins by grafting watersoluble polymers such as polyoxyethylene to some of the available amino groups of the enzyme considerably slows down the removal of the enzyme from the body (in case of PEG-asparaginase, the residence time in the body is increased from minutes to days). This is a clear practical demonstration of the importance of protein/protein interactions.

Adsorption of proteins to solid surfaces has been examined by a number of 
authors, and the results have been summarized by Andrade (1985). The hypothesis that a minimum interfacial free energy at the surface/liquid interface should lead to a minimum adsorption of "dissolved" proteins seems flawless but the experimental data do not support this theory (Coleman et al 1982). Clearly, the discrepancy is mainly due to the inability of currently available techniques and methods (e.g. contact angle measurements) to determine accurately the free interfacial energy with respect to all the components of the biological system under the relevant conditions (cf. also Andrade et al 1986). Nevertheless, even without direct experimental proof, this approach of minimizing the interfacial free energy is very attractive and is likely to lead to our being able to prepare surfaces that would not adsorb proteins (or at least the interaction with the surface would not lead to the denaturing of proteins). We would also expect such constructs to be non-immunogenic, this however depending on the changes in the constructs upon their ultimate biodegradation. Further, can we prepare surfaces that would not interact with other circulating components (e.g. platelets, erythrocytes) of the vascular compartment?

In conclusion, materials for delivering drugs to selected sites of disease within the body must be designed to utilise the unique features (structure, function, rhythm) of the main elements involved in the disease. Equally important is the design of drug carriers that do not interact non-specifically within the body such that specific action would be prevented. It is difficult to see how this could be achieved using materials foreign to the body. Utilising the primary structures used by the biological systems (proteins, glycoproteins, carbohydrates) and creating novel higher structures (secondary, tertiary, quartenary) that mimic the native material is the logical way forward in the search for new drug delivery systems, and we need to turn more and more to the molecular basis of biology for guidance and inspiration.

\section{Acknowledgements}

My thanks to Ms J Brown, Mr L Brookman and Dr P Goddard for providing me with the experimental data given in figure 6.

\section{References}

Abuchowski A, van Es T, Palczuk N C and Davis F F 1977 J. Biol. Chem. 2523578

Andrade J D (ed.) 1985 Protein adsorption (New York: Plenum)

Andrade J D and Hlady V 1986 Adv. Polym. Sci. 791

Coleman D L, Gregonis D E and Andrade J D $1982 J$. Biomed. Mater. Res. 16381

Documenta CIBA-GEIGY 19875

Margolin A L, Izumrudov V A, Sherstyuk S F, Zezin A B and Shvyadas V K 1983 Mol. Biol. 17815

Speer D P, Chvapil M, Volz R G and Holmes M D 1979 Clin. Orthop. 144326 